\title{
Análisis de las conductas de los entrenadores después del tiro en baloncesto
}

\author{
Analysis of the behavior of coaches in basketball after shot
}

\section{Análise do comportamento dos treinadores no basquete após o disparo}

\author{
Benjamín Longarela Pérez, Juan José Fernández Romero y Miguel Saavedra García \\ Facultad de Ciencias del Deporte y la Actividad Física. Universidade da Coruña.
}

\begin{abstract}
Resumen: La presente investigación analiza las conductas de los entrenadores de baloncesto después del lanzamiento a canasta (tanto de los jugadores de su equipo como del equipo contrario) en situaciones de competición en categorías de formación. Intervinieron en el presente estudio tres entrenadores, todos poseen la titulación española oficial necesaria para entrenar en las diferentes categorías. Se han analizado 9 partidos oficiales de la Liga Gallega (3 por cada uno de los 3 entrenadores participantes) y se registraron 1196 conductas tras lanzamiento. El instrumento utilizado para la recogida de datos procede de una adaptación del Coach Behavioral Assessment System (Smith, Smoll y Hunt, 1977; Cruz, 1989) y del Sistema de Observación de las Conductas del Entrenador (Montero, 2004). Tras los resultados obtenidos, se puede afirmar que las conductas de inhibición ("ignorar el error" y "no reforzar") son las más frecuentes y que el resultado en el marcador está asociado a cambios en el comportamiento de los entrenadores.

Palabras clave: datos de comportamiento, muestra, variables situacionales. Abstract: The study presented below is an analysis of the behavior of basketball coaches after the shot (players of both teams) during the competition in youth categories. In this study, three coaches participed, everybody have the official Spanish qualifications needed to train in different categories. It was analyzed 9 official games of the Galician league ( 3 each of the 3 coaches participants) and 1196 behaviors were registered after shot. The instrument
\end{abstract}

used for the data collection was an adaptation of the Coach Behavioral Assessment System (Smith, Smoll \& Hunt, 1977) and of the Sistema de Observación de las Conductas del Entrenador (Montero, 2004). After the results obtained, we can say that the inhibition behaviors (ignore mistake and not reinforcement) are the most frequently and that the score is associated with changes in the behaviors of coaches.

Keywords: behavioral data, sample, situational variables.

Resumo: O presente trabalho analisa o comportamento dos treinadores de basquete após arremesso à cesta (dos jogadores de sua equipe e da equipe adversária) em situaçóes competitivas nas categorias de formação do basquetebol. São participantes deste estudo três treinadores, todos com as certificaçôes necessárias para ser treinador em diferentes categorias na Espanha. Foram analisados nove jogos oficiais da Liga Gallega (três de cada um dos três treinadores participantes) e 1.196 observaçōes foram feitas após arremessos. O instrumento utilizado para a coleta de dados foi adaptado do Coach Behavioral Assessment System (Smith, Smoll e Hunt, 1977; Cruz, 1989) e Sistema de Observación de las Conductas del Entrenador (Montero, 2004). Como resultados, observou-se que a conduta de inibição ("ignorar erro" e "não reforçar") são os mais frequentes, e resultado no placar influencia o comportamento dos treinadores.

Palavras chave: dados comportamentais, amostra, variáveis situacionais.

\section{Introducción}

Los entrenadores deportivos son un grupo ampliamente tratado en el ámbito de la psicología del deporte (Gallimore y Tharp, 1976 y 2004; Millar, 1996; Bloom, Crumpton y Anderson, 1999; Smith y Smoll, 2009, Walters, 2011) y, dentro de estos trabajos, una línea de investigación destacada corresponde al análisis de sus intervenciones en los entrenamientos y en los partidos empleando, para ello, diversos instrumentos (Smith, Smoll y Curtis, 1979; Johnson y Franks, 1991; Miller, 1992; Smith, 1993; More, McGarry, Partridge y Franks, 1996; Trudel, Côté y Bernard, 1996; Smith y Smoll, 1997; Gilbert, Trudel, Gaumond y Larocque, 1999; Delgado Noguera, 2000; Montero, 2004). El empleo de estos instrumentos se ha orientado a objetivos psicológicos y pedagógicos, centrándose, prioritariamente, en los deportes de equipo (Rutten, Dekovic, Stams, Schuengel, Hoeksma y Biesta, 2007; González 2010). Estos deportes se caracterizan por la conjunción del esfuerzo y

Dirección para correspondencia [Correspondence address]: Benjamín Longarela Pérez. Dirección: Ronda del Carmen no $4-2^{\circ}$ derecha C.P.: 27004 Lugo (España). E-mail: b.longarela@udc.es la habilidad de diversos deportistas para alcanzar un objetivo común, mediante la consecución de goles, canastas o acciones similares que determinan el resultado de la competición.

La selección de la competición en baloncesto como ámbito de investigación, obedece a las numerosas posibilidades de interacción entre jugadores, árbitros y entrenadores que proporciona, reglamentaria y funcionalmente, esta modalidad deportiva. En este sentido, Cruz y Capdevila (1997) afirman que las respuestas de los entrenadores en situaciones de competición están condicionados por unos antecedentes e implican unas consecuencias. De este modo, sería posible comprender los diferentes comportamientos individuales, utilizando la metodología adecuada para realizar un estudio de campo, como ocurre entre los que adoptan la competición deportiva como escenario de investigación (Smith et al., 1977). Conocer las instrucciones del entrenador durante el partido y analizar su comportamiento verbal, posibilitará el establecimiento de determinados modelos de entrenador (Feu, Ibáñez, Graça y Sampaio, 2007). 
Diversos trabajos inciden en la relevancia del lanzamiento en baloncesto como predictor del resultado de los encuentros (Claramount y Balagué, 2010; Wissel, 2010), a lo que se debe añadir la importancia que le atribuyen los propios deportistas (Montero, Ezquerro y Saavedra, 2009; Ortega, Palao, Sáinz de Baranda y García, 2009); de ahí, el interés por conocer las reacciones de los entrenadores tras el lanzamiento a canasta por parte de los jugadores de ambos equipos en situaciones de competición.

Los objetivos del presente estudio son: describir las conductas de los entrenadores durante la competición deportiva tras el lanzamiento a canasta de sus jugadores y de los rivales y analizar dichas conductas en función del marcador (ganando, perdiendo o igualado).

\section{Método}

\section{Participantes}

Intervinieron en el presente estudio tres entrenadores, dos hombres y una mujer, de equipos que competían en la liga autonómica de Galicia (España) en la temporada 2009-2010: el entrenador 1, varón de 34 años de edad y 18 de experiencia, dirigía a jugadoras comprendidas entre 16 y 18 años; el entrenador 2, tenía 24 ańos, acumulaba 8 de experiencia, y dirigía a un equipo de nińos de 12 a 14 ańos; mientras que la tercera participante tenía una edad de 26 años, 8 como entrenadora, y la edad de las jugadoras de su equipo comprendía entre 14 y 16 ańos. Todos los entrenadores participantes poseen la titulación española oficial necesaria para entrenar en las diferentes categorías. Se ha solicitado y obtenido por escrito, el consentimiento de todos los sujetos participantes, así como la correspondiente aprobación del Comité de Ética de la Universidad de A Coruña (número de salida: CE 05/ 2014), para realizar el estudio.

Se han analizado 9 partidos oficiales de la Liga Gallega (3 por cada uno de los 3 entrenadores participantes en el presen- te estudio) registrándose 1196 conductas de los entrenadores tras lanzamiento.

\section{Entrevista con el entrenador}

Se realizó una entrevista estructurada con cada uno de los entrenadores antes del primer partido, siguiendo las indicaciones de Fontana y Frey (2000) y Weiss (1994), para conocer sus opiniones sobre tres bloques de contenidos: el deporte en general, el baloncesto y su equipo con especial énfasis en el lanzamiento a canasta.

\section{Instrumentos para el registro de las conductas de los entrenadores}

El instrumento utilizado para la recogida de datos procede de la adaptación española del CBAS (Smith et al., 1977) elaborado por Cruz (1989) y el del SOCE (Sistema de Observación de la Conducta del Entrenador) elaborado por Montero (2004).

Para realizar esa adaptación, se tuvieron en cuenta las posibles conductas que se podían producir tras los lanzamientos a canasta diferenciando si los jugadores encestaban o no. Para ello se consideró "acierto" cuando el lanzamiento sumaba o posibilitaba sumar puntos para el equipo del entrenador observado (por ejemplo, el lanzador recibía una falta en su acción de tiro) y, también cuando fallaban su lanzamiento los jugadores del equipo contrario. En sentido inverso, se consideró "error" cuando fallaban los jugadores de su equipo o si anotaban sus lanzamientos los jugadores del equipo contrario. La variable "marcador" se categorizó, siguiendo el criterio de Montero (2004), como "ganando" cuando el lanzamiento se producía con una ventaja superior o igual a 4 puntos para el equipo analizado, "igualado" si la diferencia en el marcador no superaba 3 puntos y "perdiendo", si la desventaja en el marcador era de 4 o más puntos en función de que en baloncesto, con un único lanzamiento se pueden conseguir hasta tres puntos.

Las categorías se definieron como se describen en la tabla 1.

Tabla 1. Categorías empleadas en el estudio y definición de las mismas.

\begin{tabular}{lll}
\hline Positivas & 1.- Refuerzo tras acierto & Conducta de elogio o reconocimiento tras un acierto (ej., gran tiro, bien). \\
\cline { 2 - 3 } & 2.- Ánimo tras error & Un jugador lanza y falla y el entrenador lo anima (ej., no pasa nada). \\
\cline { 2 - 3 } & 3.- Instrucción técnica tras error & $\begin{array}{l}\text { Tras un error el entrenador indica, lo que tendría que hacer en esa situación (ej., flexiona } \\
\text { las rodillas). }\end{array}$ \\
\hline Negativas & 4.- Castigo & El entrenador emite una conducta punitiva tras un error (ej., iqué mal!). \\
\cline { 2 - 3 } & 5.- Instrucción técnica punitiva & $\begin{array}{l}\text { El entrenador tras un error en la acción de tiro, le señala lo que debería hacer, en forma } \\
\text { de castigo (ej., jno quiero esos tiros cuando van } 10 \text { segundos de posesión!). }\end{array}$ \\
\hline Inhibición & 6.- No refuerzo tras acierto & $\begin{array}{l}\text { No se aprecia ninguna conducta observable tras un acierto de su equipo, o fallo del } \\
\text { equipo contrario. }\end{array}$ \\
\cline { 2 - 3 } & 7.- Ignora error & $\begin{array}{l}\text { Tras un fallo de algún jugador de su equipo, o acierto del equipo contrario, el entrena- } \\
\text { dor no manifiesta ninguna conducta observable. }\end{array}$ \\
\hline Otras & 8.- Otras conductas tras acierto & Se produce una conducta distinta a las anteriores tras un acierto (ej., jdeberías penetrar!). \\
\cline { 2 - 3 } conductas & 9.- Rebote & Tras un lanzamiento de cualquier jugador, el entrenador grita "rebote”. \\
\hline
\end{tabular}




\section{Entrenamiento de los observadores}

Dos licenciados en Ciencias de la Actividad Física y el Deporte, expertos en baloncesto, participaron en este estudio, completando las tareas de entrenamiento que se realizaron en tres fases, con una duración aproximada de 50 horas, hasta alcanzar un grado de acuerdo mínimo de 0,80 según el índice de concordancia Kappa de Cohen.

\section{Instrumentos utilizados para el registro de la conducta}

Cámaras de vídeo, cintas de vídeo y sonido

Para la grabación de los partidos se utilizaron dos cámaras de vídeo digitales, mientras que el sonido se grabó mediante un micrófono de solapa que se le proporcionaba al entrenador antes de cada partido.

Hojas de registro

Para la codificación y registro de las variables consideradas en el presente estudio, se diseñó una hoja que contempla: el tipo de lanzamiento, el número del jugador, la conducta del entrenador, el momento en que se emite dicha conducta, a quien se dirige el entrenador y el marcador en el momento de efectuarse el lanzamiento.

\section{Procedimiento estadístico}

Se presentan tablas de frecuencias (absolutas y relativas) de los distintos tipos de conductas mostradas por los entrenadores.

Para comprobar si existen diferencias significativas entre las conductas positivas, negativas, de inhibición y otras conductas de los entrenadores, se ha utilizado la prueba de Kruskal-Wallis mediante la cual se han determinado diferencias estadísticamente significativas entre las conductas de los entrenadores $(\mathrm{p}<0.001)$.

\section{Resultados}

Del análisis de los 9 partidos oficiales observados se derivan los siguientes resultados sobre las conductas de los entrenadores tras lanzamientos a canasta de su equipo y del equipo rival.

\section{Conductas observadas de cada entrenador}

En la tabla 2 se presentan los valores de las conductas de los entrenadores tras lanzamientos del equipo al que entrena y tras lanzamientos del equipo rival.
Tabla 2. Frecuencias absolutas y relativas de las conductas de los entrenadores tras lanzamientos del equipo al que entrena y del equipo rival.

\begin{tabular}{lccc}
\hline Conductas & $\begin{array}{c}\text { Tras lanzamiento } \\
\text { equipo propio }\end{array}$ & $\begin{array}{c}\text { Tras lanzamiento } \\
\text { equipo rival }\end{array}$ \\
\hline \multirow{3}{*}{ Positivas } & (tras acierto) & $83(13,8)$ & $\mathrm{n}(\%)$ \\
& (tras error) & $46(7,6)$ & $0(0)$ \\
\hline
\end{tabular}

\begin{tabular}{rcc} 
Negativas & $2(0,3)$ & $2(0,4)$ \\
\hline Inhibición (tras acierto) & $183(30,4)$ & $307(51,6)$ \\
(tras error) & $279(46,4)$ & $275(46,2)$ \\
\hline
\end{tabular}

$\begin{array}{rcc}\text { Otras conductas } & 8(1,3) & 10(1,6) \\ \mathrm{N}=1.196 & 601 & 595\end{array}$

Como se puede observar en la tabla 2, las conductas más habituales de los entrenadores tras los lanzamientos de ambos equipos son las de inhibición, que alcanzan su valor más elevado tras aciertos del equipo rival. Por su parte, las conductas positivas se producen únicamente, tras lanzamientos de los jugadores de su equipo. Del total de estas conductas, las registradas tras acierto doblan a las que se produjeron tras algún fallo en el lanzamiento de sus jugadores; por último destacar que las conductas negativas presentan valores muy bajos.

Descripción de las conductas de los entrenadores en función del marcador

En la tabla 3 se presentan los datos de las conductas de los entrenadores, en función del marcador (ganando, igualado o perdiendo) cuando se produce el lanzamiento.

Tabla 3. Frecuencias absolutas y relativas de las conductas de los entrenadores en relación al marcador.

\begin{tabular}{lcccc}
\hline Conductas & $\begin{array}{c}\text { Ganando } \\
\mathrm{n}(\%)\end{array}$ & $\begin{array}{c}\text { Igualado } \\
\mathrm{n}(\%)\end{array}$ & $\begin{array}{c}\text { Perdiendo } \\
\mathrm{n}(\%)\end{array}$ & $\begin{array}{c}\text { Significa- } \\
\text { ción* }\end{array}$ \\
\hline Positivas & $70(12,6)$ & $35(14,8)$ & $25(6,2)$ & \\
Negativas & $4(0,7)$ & $0(0)$ & $0(0)$ & 0,001 \\
Inhibición & $473(84,9)$ & $199(84,3)$ & $372(92,3)$ & \\
Otras conductas & $10(1,8)$ & $2(0,8)$ & $6(1,5)$ & \\
$\mathrm{N}=1.196$ & 557 & 236 & 403 & \\
\hline
\end{tabular}

*Significación de la prueba de Kruskal-Wallis

Como se puede observar, las conductas con mayor porcentaje en cualquier situación del marcador son las de inhibición; seguidas por las conductas positivas, que muestran un incremento si el marcador está igualado; mientras que las conductas negativas, únicamente se registran cuando el marcador es 
favorable. La prueba de Kruskal-Wallis muestra diferencias estadísticamente significativas entre los grupos de conductas en función del marcador.

\section{Discusión}

Las conductas más habituales de los entrenadores analizados corresponden a las categorías de inhibición $(76,9 \%$ tras lanzamientos de su equipo y $97,8 \%$ tras tiros del rival); valores superiores al 59\% y 86,5\% obtenidos por Montero (2004), aunque, por su parte Mowat (2004), únicamente registró un $4,89 \%$. Estas diferencias en los resultados pueden deberse a que la presente investigación y los datos de Montero (2004) se refieren únicamente a las conductas tras lanzamientos a canasta, mientras que Mowat (2004) analiza todas las acciones de los jugadores en la competición.

El segundo grupo más frecuente es el de conductas "positivas" (21,4 \% tras lanzamientos de su equipo, valor inferior al indicado por Walters (2011) quien registró un 35,4\% de comentarios de apoyo en entrenadores de rugby, tenis y fútbol y a los de Cruz et al., 1987), quienes señalan un 64,6\% en baloncesto infantil. Esta diferencia en los resultados entre Cruz et al., (1987) o Walters (2011) y el presente estudio, podría deberse a la evolución en la formación de los entrenadores quienes, a lo largo del tiempo, han ido adquiriendo competencias y conocimientos que les permiten centrarse además en diversos aspectos técnicos, tácticos y de estilo de comunicación durante la competición.

Dado que las conductas positivas únicamente se producen tras lanzamientos de los jugadores de su equipo, podría indicarse que los entrenadores se centran en su equipo y los jugadores que dirige obviando, incluso, los errores en el lanzamiento por parte del equipo rival.

Las conductas positivas registradas en la presente investigación representan un 21,7\% y las negativas un $0,7 \%$, lo que revela que las conductas de apoyo fueron mucho más frecuentes; datos similares a los de Millard (1996), en su estudio en fútbol escolar, que registró un $42 \%$ de conductas de apoyo frente a un 7\% de negativas. La interpretación de las diferencias entre los deportes analizados, podrían referirse a que el número de lanzamientos, el contexto en el que se producen y las consecuencias para el resultado de la competición deportiva pueden variar sustancialmente de una modalidad a otra.

Al considerar el resultado, es posible apreciar que las con- ductas de inhibición son las más frecuentes, independientemente de lo que refleja el marcador, misma tendencia que la registrada por Trudel (1997) en su estudio a entrenadores de hockey sobre hielo y Montero (2004). Por su parte, las conductas positivas son más frecuentes si el marcador es igualado, mientras que Montero registró que dichas conductas se incrementan a medida que el resultado se convierte en positivo. Los valores reseńados permiten suponer un peso reducido de las conductas negativas y un incremento progresivo de las positivas a medida que se iguala el marcador en los entrenadores de deportes de equipo en categorías de formación. Las diferencias registradas entre Montero (2004) y el presente estudio pueden deberse a las adaptaciones realizadas en el instrumento utilizado para el registro y análisis de las conductas así como a la edad y experiencia de los entrenadores implicados.

De los resultados obtenidos en el presente estudio, se puede afirmar que las conductas más frecuentes de los entrenadores participantes son las de inhibición. El segundo grupo de conductas más frecuentes son las positivas, que se producen únicamente tras lanzamientos de los jugadores de su equipo, puesto que no refuerzan los errores en el lanzamiento del equipo contrario; en síntesis, los entrenadores se centran casi exclusivamente en los jugadores de su equipo.

Por último, tras el análisis de las conductas del entrenador en función del marcador, las conductas positivas se incrementan a medida que se iguala el resultado, mientras que las negativas sólo se producen cuando la situación en el marcador es favorable y en una proporción muy baja.

\section{Aplicaciones prácticas}

Desde un punto de vista práctico, resulta fundamental para nuestro deporte dotar a los entrenadores de toda la información posible para mejorar en su proceso de formación y para conseguir que los jugadores reciban la mejor atención posible. De esta manera, el presente estudio pone de manifiesto la importancia de las conductas de los entrenadores, o sea, qué dice, cómo lo dice y las consecuencias que eso tiene en la satisfacción y continuidad de los deportistas.

Resulta fundamental que dichos deportistas reciban la mejor formación posible y para ello, es necesario que los entrenadores tengan conocimiento sobre cómo dirigirse a ellos y así poder modificar, incrementar o reducir determinadas conductas.

\section{Referencias}

1. Bloom,G., Crumpton, R. y Anderson, J. (1999). A Systematic Observation Study of the Teaching Behaviors of an Expert Basketball Coach. The Sport Psychologist, 13, 157- 170.

2. Claramount, C. y Balagué, N. (2010). Influencia de las instrucciones técnicas en la efectividad del lanzamiento en baloncesto. Apunts. Educación Física y Deportes, 99, 65-71.
3. Cruz, J. (1989). Proyecto de Investigación: Influencia del entrenador en la motivación de deportistas jóvenes: su evaluación y su cambio. Universidad Autónoma de Barcelona: Barcelona.

4. Cruz, J., Bou, A., Ferrández, J., Martín, M., Monrás, J., Monfort, N. y Ruiz, A. (1987). Avaluació conductual de les interaccions entre entrenadors i jugadors de bàsquet escolar. Apunts. Medicina de l'Esport, 24, 89-98. 
5. Cruz, J. y Capdevila, Ll. (1997). Evaluación en psicología del deporte. En G. B. Casal y J. C. Sierra (eds.) Manual de evaluación psicológica. (pp. 924-947). Fundamentos, técnicas y aplicaciones. Madrid: Siglo XXI.

6. Delgado Noguera, M. (2000). Análisis de los comportamientos de los docentes en la enseñanza deportiva en edad escolar. Actas del Congreso Nacional De Deporte En Edad Escolar; Dos Hermanas: Sevilla, 17, 18 y 19 de Noviembre de 2000.

7. Feu, S., Ibáñez, S., Graça, A. y Sampaio, J. (2007). Evaluación psicométrica del cuestionario de orientación de los entrenadores en una muestra de entrenadores espańoles de balonmano.Psicothema, 19 (4), 699-705.

8. Fontana, A. y Frey, J. (2000). The interview. From structured questions to negotiated text. En N. K. Denzin e Y.S. Lincoln (Eds.) Handbook of Qualitative Research (pp.645-672). Thousand Oaks: Sage.

9. Gallimore, R. y Tharp, R. (2004). What a Coach Can Teach a Teacher, 1975-2004: Reflections and Reanalysis of John Wooden's Teaching Practices. The Sport Psychologist, 2 (18), 119-137.

10. Gilbert, W., Trudel, P., Gaumond, S. y Larocque, L. (1999). Development and application of an instrument to analyse pedagogical content interventions of ice hockey coaches. Sociology of Sport On-line, 2 (2). http://physed.otago.ac.nz/sosol/v2i 2/v2i2a2.htm

11. González, Ma . (2010). Evaluación psicológica en el deporte: aspectos metodológicos y prácticos. Papeles del Psicólogo, 3 (31), 250-258.

12. Johnson, R. y Franks, I. (1991). Measuring the reliability of a computer-aided systematic observation instrument. Canadian journal of Sport Science, 16, 45-57.

13. Millard, L. (1996). Differences in coaching behaviours of male and female high school soccer coaches. Journal of Sport Behavior, 19 (1), 19-31.

14. Miller, A. (1992). Systematic observation behavior similarities of various youth sport soccer coaches. The Physical educator, 49, 136-143.

15. Montero, A. (2004). Evaluación de las conductas del entrenador de baloncesto infantil. Tesis doctoral. Universidade da Coruña, A Coruña.

16. Montero, A., Ezquerro, M. y Saavedra, M. (2009). Application of a token economy program and preferences for basketball players. Revista de Psicología del Deporte, 18 (3), 433-437.

17. More, K., Mcgarry, T., Partridge, D. y Franks, I. (1996). A computer- assisted analysis of verbal coaching behavior in soccer. Journal of Sport Behavior, 19, 319-337.

18. Mowat, J. (2004). Arousal and behaviour of coaches during competition. Tesis doctoral. Faculty of Human Development, Victoria University of Technology.

19. Ortega, E., Palao, J., Sáinz de Baranda, P. y García, L. (2009). Preferences and levels of satisfaction in technical and tactical actions and in type of offense and defense utilized in competition by youth basketball players. Revista de Psicología del Deporte, 18 (3), 343-348.

20. Smith, R. (1993). Intervenciones psicoeducativas en el deporte. Madrid: Pirámide.

21. Smith, R. y Smoll, F. (1997). Coaching the coaches: youth sports as a scientific and a applied behavioural setting. Current Directions in Psychological Science, 6 (1), 16-21.

22. Smith, R. y Smoll, F. (2009). Claves para ser un entrenador excelente. Barcelona: INDE.

23. Smith, R., Smoll, F. y Curtis, B. (1979). Coach effectiveness training: A cognitive- behavioral approach to enhancing relationship skills in youth sport coaches. Journal of Sport Psychology, 1, 59-75.

24. Smith, R., Smoll, F. y Hunt, E. (1977). A system for the behavioural assessment of athletic coaches. Research Quaterly, 48 (2), 401-407.

25. Tharp, R. y Gallimore, R. (1976). Basketball's John Wooden: Wath a coach can teach a teacher. Psychology Today, 9 (8), 74-78.

26. Trudel, P. (1997). L' influence des periódes de jeu et de l'écart au store sur les comportements des entrenaïeurs lors de matchs au jockey sus glace. STAPS, no 43, Junio, 71-74.

27. Trudel, P., Côté, J. y Bernard, D. (1996). Systematic observation of youth ice hockey coaches during games. Journal of Sport Behavior, 19 (1), 50-65.

28. Walters, S. (2011). Whose Game are we playing? A study of the effects of adult involvement on children participating in organised team sports. Tesis doctoral.Auckland University of Technology.

29. Weiss, R. (1994). Learning from strangers. The art and method of qualitative interview studies. New York: The Free Press.

30. Wissel, H. (2010). Técnicas de tiro. Cap.1. En Giorgio Gandolfi (Dir.) Libro de jugadas de los entrenadores de la NBA (pp. 25-41). Madrid: Tutor. 